\title{
Essai d'analyses de la conception paysanne de l'évolution, de la gestion et de l'utilité du Parc National de Mozogo-Gokoro (Cameroun) en vue de son aménagement
}

\author{
Rodrigue Constant SANDJONG SANI ${ }^{1 *}$, Mama NTOUPKA ${ }^{2}$, Adamou IBRAHIMA ${ }^{3}$ et \\ Toua VROUMSIA ${ }^{4}$

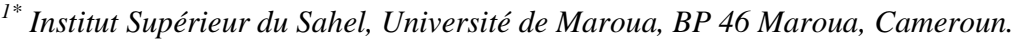 \\ ${ }^{2}$ IRAD Forêt - BP 222 Maroua, Cameroun. \\ ${ }^{3}$ Université de Ngaoundéré, Faculté des Sciences, Département de Sciences Biologiques, \\ B.P. 454 Ngaoundéré, Cameroun. \\ ${ }^{4}$ Université de Maroua, Ecole Normale Supérieure, Département de SVT, BP 55 Maroua, Cameroun. \\ *Auteur correspondant, E-mail: sanirocos@yahoo.fr , Tel. (+237)75069143/(+237)94827869
}

\section{RESUME}

Cet essai d'exploration du milieu humain riverain du Parc National de Mozogo-Gokoro est une démarche visant sa conservation et son aménagement, en exploitant des indicateurs facilement compréhensibles. Une enquête socio-économique à orientations écologiques a été réalisée afin d'apprécier la perception paysanne de l'évolution spatio-temporelle, compositionnelle et structurale du parc, de son utilité, et de sa gestion avec confrontation des résultats à la vision de l'administration forestière locale. Les résultats ont montré que c'est un écosystème qui a subi une forte pression anthropique, et l'effet de la sècheresse avec une trajectoire régressive. Plusieurs espèces animales et végétales sont signalées en disparition ou en diminution dans le Parc, reflétant un recul de la biodiversité. Son utilité est illustrée par la diversité des ressources (Produits Forestiers Non Ligneux, bois de service et d'énergie) prélevées ou dont le besoin a été exprimé par la population riveraine, indiquant par ailleurs une gestion non conforme à la réglementation. Des orientations d'aménagement, découlant de cette perception, ont été proposées afin d'atténuer la dynamique régressive amorcée et causée par l'absence d'un plan de gestion participative, renforçant sa protection intégrale.

(C) 2013 International Formulae Group. All rights reserved.

Mots clés : Enquête, dynamique spatio-temporelle, perception, population, végétation, protection.

\section{INTRODUCTION}

La mise en œuvre intégrale des instruments juridiques internationaux pour une gestion durable des ressources naturelles s'est parfois butée à des contextes géographiques et nationaux plus ou moins réfractaires à ces mutations. Dans les régions sahéliennes et soudaniennes, les facteurs mis en cause sont notamment climatiques (épisodes de sècheresses) et humains (défrichements, coupes de bois, surpâturages, feux incontrôlés, élevage transhumant), Dupuy et al. (1999), COMIFAC/CEEAC (2007), ECODIT (2008).

Le gouvernement camerounais se préoccupe de ces aires dites à écologie fragile 
situées dans sa partie septentrionale. Une grande vitalité de programmation liée à une législation forestière de gestion durable des forêts est observée à l'instar du Programme Sectoriel Forêt Environnement. Toutefois, des problèmes sont signalés dans l'application de cette politique forestière (Nke Ndih, 2008 ; UICN, 2009). Ils sont relatifs à la traçabilité des activités d'extraction et de partage des ressources, les droits d'accès de cette ressource aux acteurs non professionnels, particulièrement la population locale, et la sécurisation des droits acquis de par la loi (UICN, 2009). Cette situation dérive d'une gestion sans plan d'aménagement de plusieurs espaces forestiers. Différentes aires protégées de la région administrative camerounaise de l'Extrême-Nord sont concernées, spécifiquement notre site d'étude: le Parc National de Mozogo-Gokoro (Donfack, 2009). La FAO, lors de la dixième session du Comité de mise en valeur des forêts tropicales en décembre 1992 présente l'aménagement durable sous deux angles: la planification et l'exécution d'actions destinées à assurer la conservation et l'utilisation d'une forêt en fonction d'objectifs, du contexte physique et socio-économique, la satisfaction des besoins des générations actuelles et futures pour un développement durable (Bellefontaine, 2005). Dans ce contexte, les règles d'accès et les pratiques liées à la gestion des ressources constituent un enjeu important et passent nécessairement par une meilleure connaissance des ressources et des besoins des différents acteurs (Ntoupka et al., 2006). Des enquêtes de terrain, orientées vers le milieu humain en constituent donc l'un des préalables. Elles représentent une source d'informations compréhensibles par un bon nombre de décideurs. Notre travail se situe aussi, dans un processus de suivi écologique, préconisé pour la gestion efficace et durable des aires protégées, avec implication de la population riveraine. Nguinguiri (1998) révèle l'inefficacité de la conservation des ressources forestières au moyen de pratiques qui visent à exclure la population, avec pour conséquences beaucoup d'incompréhensions et des conflits liés ou sentiment de confiscation des ressources forestières par l'État. Les approches participatives de gestion des ressources naturelles ont été ces dernières années très fortement soutenues par les bailleurs et les institutions en charge des programmes de conservation de la biodiversité (Hulme et Murphree, 2001; Adams et al., 2004 ; Robbins et al., 2006, cités par Binot et Joiris, 2007). Dans cette visée, les détenteurs $\mathrm{du}$ pouvoir de décision en matière de management de ces ressources doivent s'appuyer sur la population locale avec pour objectif, qu'elle ait un grand bénéfice des efforts de conservation fournis (Van Der Breemer et al., 1995, cités par Halidou, 2000).

L'objectif global est d'établir à partir de l'opinion de la population riveraine, des actions favorables à la conservation et l'aménagement de cette aire protégée. Plus spécifiquement, il s'agit d'obtenir de la population locale, des informations personnelles permettant de la décrire brièvement en rapport au contexte, d'analyser son appréciation de l'évolution spatiotemporelle, compositionnelle et structurale de la végétation du parc, de sa gestion et son utilité, et de montrer l'urgence d'une intervention par des orientations d'aménagement découlant de cette perception.

\section{MATERIEL ET METHODES}

\section{Généralités sur le site d'étude}

Le site d'étude est situé au niveau des piémonts des monts Mandara, dans la plaine de Koza, entre $10^{\circ} 56^{\prime}$ et $10^{\circ} 96^{\prime}$ de latitude Nord et $13^{\circ} 54^{\prime}$ et $13^{\circ} 58^{\prime}$ de longitude Est. D'une superficie de 1400 hectares (MINEF, 2000), il a été créé comme réserve forestière et de faune par arrêté $\mathrm{N}^{\circ}$ 165 du 12 Juin 1932 du Haut Commissaire de 
la République française au Cameroun et, classé en parc national par arrêté $\mathrm{N}^{\circ} 120$ du 05 décembre 1968 du Secrétariat au Développement de l'Etat du Cameroun (Wafo, 2008).

La carte établie par Brabant et Gavaud (1985) indique la présence, dans la zone, des sols évolués tels que les planosols et solonetz gleyiques sur granite d'anatexie et Gneiss, des sols peu évolués exondés des alluvions anciens des cours d'eau temporaires (Mayo) ainsi que des sols inondés de plaine de décantation argileuse (vertisols hydromorphes). Le climat est de type soudano-sahélien avec une pluviosité comprise entre 600 et $900 \mathrm{~mm}$ (Yann, 1998).

Letouzey (1985) décrit la végétation de la région d'étude comme étant située dans le vaste ensemble constitué par les monts Mandara, et composée de savanes arbustives médio-soudaniennes à Isoberlinia doka (faciès dégradés), de savanes arbustives à arborées à Anogeissus leiocarpus dégradées à épineux dominants et de savanes arbustives à Anogeissus leiocarpus dégradées. Cependant, considérant les caractéristiques géomorphologiques de notre site situé en plaine et d'autres informations du même auteur concernant les limites floues des zones éco-géographiques, la végétation peut être qualifiée de sahélo-soudanienne ou soudanosahélienne, savanicole ou steppique, avec plusieurs faciès de dégradation. La faune observée dans le parc de façon très récente est constituée de plusieurs groupes de Mammifères (Artiodactyles, Primates, Rongeurs, Carnivores...), de nombreux Reptiles, une avifaune variée (114 espèces), des Batraciens et Invertébrés (MINEF, 2000).

C'est une zone densément peuplée. La population, organisée autour des sept grandes contrées riveraines, appartient à cinq grands groupes ethniques: les Mafa majoritaires, les Mandara, les Kanuri, les Glavda et les Peuls. Une redistribution majeure de population est intervenue, dans cette région, ces dernières années, avec la colonisation de la plaine liée à la stabilité politique et à la mise en place des structures sociales incitatives (établissements scolaires, hôpitaux...). Ceci établit, de fait, une forte pression foncière, avec mise en culture des nouvelles terres, diminution et disparition des jachères (Zwiderwijk et Wouter, 2000). Cette menace anthropique est perceptible dans la plaine de Koza, avec de probables répercussions au niveau du parc.

\section{Méthodes}

Une enquête par questionnaire structuré, avec possibilité de reformulation en cas d'incompréhensions a été menée, auprès de la population riveraine ainsi qu'auprès de deux anciens et du conservateur en poste, par souci de confrontation. Elle a reposé sur des informations personnelles, sur la perception de l'évolution dans le temps et l'espace du parc, de sa gestion et de son utilité, afin de formuler par la suite quelques propositions d'aménagement dans le respect des principes de cogestion. Des critères tels que l'âge et le sexe sont considérés dans l'échantillonnage. Tous les villages périphériques sont concernés (Figure 1) dans la collecte des données, soit un nombre de 5 personnes pour chaque contrée: Mawa, karazawa, Gabas, Gokoro, Nguétchéwé, Yamgazawa et Mozogo et 3 supplémentaires pour la dernière citée plus peuplée. La traduction de certains noms vernaculaires a été possible en utilisant le document de Malzy (1954). Ces données socio-économiques sont synthétisées et analysées dans la mesure du possible par des tests statistiques. 


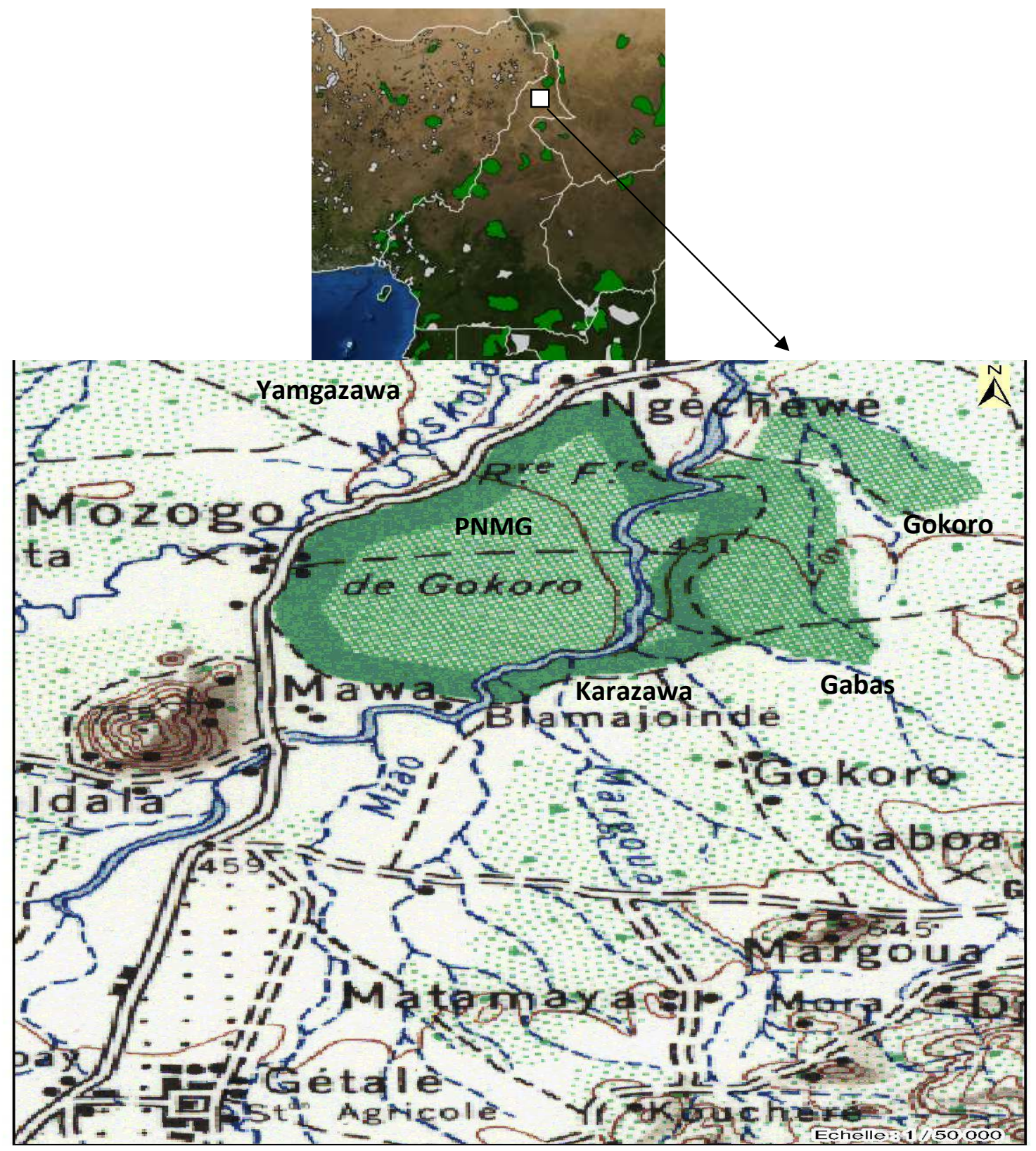

Figure 1 : Carte représentant le Parc National de Mozogo-Gokoro (PNMG) avec localisation des sept contrées périphériques (Adaptation de la carte de la SACART, 2008).

\section{RESULTATS}

Environnement humain du PNMG relatif à l'échantillonnage

Les résultats de notre enquête prouvent la diversité des utilisateurs probables des ressources du parc présents dans la population riveraine. Tenant compte de la taille moyenne des ménages estimée à 9 individus $(8,7 \pm 1,4)$, le nombre de personnes concernées par l'enquête, et dont les opinions peuvent se rallier à celles des interviewés est de 405. L'agriculture est la première occupation des 
populations riveraines du parc $(53,33 \%$ de ménages). Elle est souvent associée au commerce $(11,11 \%)$, à l'élevage sédentaire de bovins, surtout de caprins et d'ovins $(15,56 \%)$ ou la médecine traditionnelle $(6,67 \%)$. Les femmes représentent $8,88 \%$ de notre échantillon. L'âge moyen des personnes enquêtées est de 49,7 $\pm 8,4$ ans. Le constat qui se dégage est que $64,33 \%$ d'individus ont une ancienneté dans les localités, supérieure ou égale à 30 ans, suffisante pour percevoir l'évolution du parc.

\section{Perception de l'évolution spatio-temporelle, compositionnelle et structurale de la végétation}

$70,18 \%$ de personnes ont affirmé connaître les limites du parc et sont donc capables d'apprécier l'évolution de la superficie et la structure de la végétation.

Le Tableau 1 donne une vue de la perception villageoise de l'évolution de la superficie et la structure de la végétation. Des tableaux de contingence sont construits à partir de ces valeurs pour l'application du test d'indépendance. Il permet d'affirmer d'une part, que la superficie du parc a changé avec le temps, avec une valeur de $\mathrm{X}^{2}=11,009$ et une probabilité très faible se situant entre $[0,001 ; 0,01]$ et, d'autre part, la dynamique structurale (densité et taille des arbres) perçue en augmentation est moins probable (probabilité située entre $[0,5 ; 0,9]$ et $\mathrm{X}^{2}=$ 0,038 ) que celle en diminution (probabilité > 0,9 et $\mathrm{X}^{2}=0,010$ ) ou en stabilité (probabilité $\left.>0,9 \mathrm{X}^{2}=0,012\right)$. Cependant, le rapprochement des probabilités montre une appréhension assez confuse des changements observables dans la végétation du parc. Outre cela, la population se fait une idée plus ou moins précise des espèces animales ou végétales ayant diminué, augmenté ou disparu dans le parc et ses environs. Le Tableau 2 nous donne une idée de cette perception. L'analyse de ce tableau indique la tendance régressive de la biodiversité animale et végétale du parc. Les espèces animales relevées se rangent dans les grands groupes suivants : Mammifères et Reptiles, en majorité déjà signalées dans la littérature à l'exception de celles considérées comme disparues (lion, éléphant, buffle, girafe, hyène, léopard, singe rouge). $70 \%$ des végétaux cités appartiennent au groupe des ligneux. Dix sept de ces espèces ligneuses citées n'ont pas été inventoriées dans l'étude floristique préliminaire (Sandjong, 2012). Elles sont répertoriées dans le Tableau 3.

\section{Gestion et utilité du parc}

Les résultats de notre enquête nous indiquent une forte pression anthropique capable d'affecter négativement la végétation du parc (Figure 2). Les feux de brousse sont beaucoup plus signalés dans le passé et en bordure de l'aire protégée. D'autres menaces sur la stabilité du parc et des insuffisances dans la gestion sont témoignées par plusieurs opinions villageoises comme le confirme le Tableau 4.

Tous les villages sont impliqués dans les prélèvements illégaux de ressources. Ces derniers ont lieu surtout en saison sèche, en raison des facilités d'accès, liées à l'existence de multiples pistes, en plus des $20 \mathrm{~km}$ aménagées par l'administration. Pour la ressource en bois, plus convoitée, les mécanismes de prélèvement ne sont pas toujours compatibles avec la régénération et la croissance faciles (Figure 3). Les fréquences de prélèvement se situent au-delà de 8 fois en moyenne par mois et par ménage. Malgré quelques problèmes soulevés et relatifs à la destruction des cultures par les Babouins et les Oiseaux granivores, le parc est d'une importance capitale pour les riverains. Un rôle de production découle incontestablement du 
prélèvement ou de l'expression de besoins en ressources diversifiées (Tableau 5).

\section{Vision de l'administration forestière locale}

D'après les conservateurs ayant exercé dans le parc ces 15 dernières années, l'aire protégée présente des limites précises, du moins depuis son bornage en 1994. Ils ajoutent, par ailleurs, que l'inexistence depuis sa création d'une zone tampon aurait entraîné l'empiétement sur la superficie actuelle. Ils analysent aussi une dynamique régressive de la végétation et de modifications floristique et faunique profondes en accord avec la perception paysanne. Cependant, la connaissance limitée des ressources disponibles et l'appréciation divergente de l'évolution de plusieurs espèces peuvent être notées, ainsi que l'information selon laquelle à l'exception de la zone de Gabas et quelques montagnes périphériques, les environs du parc sont cultivés et non susceptibles d'abriter de façon permanente la faune sauvage.

Ces gestionnaires forestiers locaux réaffirment également l'exploitation illégale des ressources du parc (Bois et Produits Forestiers Non Ligneux), malgré l'aveu difficile qu'elle soit parfois autorisée en violation de la réglementation. Ils ajoutent aussi que tous les villages sont concernés et que les prélèvements se font surtout pendant la saison sèche, d'octobre à juin, à l'aube, au crépuscule ou pendant la nuit. Pour être plus précis, s'agissant du braconnage, des coups de feu sont entendus aux heures tardives dans la nuit, des traces de feux de chauffage et des points de cachette sont observés aux abords des mares artificielles créées. Ils précisent par ailleurs que les feux de brousse sont relativement absents, parfois observés à la lisière et très vite maîtrisés.

Ces acteurs de la gestion forestière interviewés évoquent aussi un certain nombre de problèmes rencontrés dans la gestion du parc, surtout relatifs à l'absence d'un plan d'aménagement recommandé par le décret $\mathrm{N}^{\circ}$ 95/466/PM du 20 Juillet 1995 de la République du Cameroun, fixant les modalités d'application du régime de la faune et des forêts au Cameroun.

\section{Orientations d'aménagement}

De l'analyse de nos résultats et en s'inspirant de la démarche proposée par plusieurs auteurs pour la conception des plans d'aménagement des forêts naturelles des zones tropicales sèches (Dupuy et al., 1999 ), les orientations d'aménagement ci-après sont formulées, avec pour objectifs principaux d'améliorer la gestion du parc, de renforcer sa protection et de faciliter son suivi écologique à travers les recherches.

Dans un premier temps, l'état doit mettre en place un cadre de concertation impliquant les différents acteurs pour une gestion durable et participative du parc avec exploitation de toutes les données disponibles dans ce processus, surtout dans un contexte menaçant avec le grand afflux dans la zone de populations réfugiées venues du Nigeria voisin. La sensibilisation de la population riveraine, ainsi que les autres principaux acteurs de la gestion, doit s'effectuer ensuite pour faire comprendre les enjeux de la conservation. Elle doit également insister sur la responsabilité de l'Homme dans l'évolution de la végétation, les droits d'usage dans la législation forestière et les modes de prélèvement des ressources, respectueux de l'environnement, par facilitation de la régénération et la croissance végétale. La perception globale par cette dernière de l'évolution régressive des espèces animales et végétales, est un critère important de jugement des modifications sensibles affectant la végétation à exploiter au cours de cette éducation environnementale. Au bout du compte, il faut un bon compromis entre 
l'épanouissement de la population locale et la préservation de l'environnement.

La délimitation d'une zone tampon, incluant les zones agricoles et les montagnes périphériques s'avère nécessaire pour l'application des principes de cogestion et l'exercice des droits d'usage. Considérant les besoins en ressources (bois et PFNL) exprimés par la population locale, il peut être développé, dans cette zone, des activités alternatives agro-sylvo-pastorales génératrices de revenus. Le renforcement des mesures de protection peut se faire par l'implantation de haies vives à certains endroits où l'accès est facile. L'organisation de patrouilles ou contrôles nocturnes, surtout en saison sèche est vivement recommandée. En cas d'infractions, des sanctions prévues par la réglementation en vigueur doivent être appliquées.
La projection d'activités de recherche approfondie doit débuter par une bonne caractérisation écologique, afin de mieux cerner les attributs vitaux conférant à la végétation du parc le statut d'écosystème de référence en zone sahélo-soudanienne (Sandjong, 2012).

Le suivi écologique spatio-temporel, donnant des explications sur la dynamique des espèces et la maîtrise des facteurs de dégradation, peut être entrepris pour faciliter les mesures de restauration en relation avec la surexploitation ou la disparition de ces dernières. De façon spécifique, concernant la faune, l'accent sera mis sur l'amélioration de la qualité de l'habitat en relation avec certaines exigences de la population riveraine, notamment la préservation de leurs cultures et récoltes.

Tableau 1 : Appréciation villageoise de l'évolution de la superficie et la structure de la végétation du parc par rapport aux années 80 et 90 du siècle passé.

\begin{tabular}{lcccccc}
\hline Appréciation & \multicolumn{2}{c}{ En diminution } & \multicolumn{2}{c}{ En augmentation } & \multicolumn{2}{c}{ Egalité ou stabilité } \\
\cline { 2 - 7 } & Années & Années & Années & Années & Années & Années \\
& $\mathbf{8 0}$ & $\mathbf{9 0}$ & $\mathbf{8 0}$ & $\mathbf{9 0}$ & $\mathbf{8 0}$ & $\mathbf{9 0}$ \\
\hline Superficie & 11 & 22 & 18 & 17 & 11 & 3 \\
Densité de la végétation & 21 & 10 & 17 & 17 & 4 & 4 \\
Taille de la végétation & 20 & 9 & 21 & 23 & 11 & 10 \\
(évolution des strates) & & & & & & \\
\hline
\end{tabular}

Tableau 2 : Perception paysanne de l'évolution des espèces animales et végétales.

\begin{tabular}{lcccccc}
\hline & \multicolumn{3}{c}{ Espèces animales } & \multicolumn{3}{c}{ Espèces végétales } \\
\cline { 2 - 7 } & A & B & C & A & B & C \\
\hline Nombre d'espèces & 29 & 49 & 37 & 37 & 59 & 50 \\
\hline $\begin{array}{l}\text { Pourcentage d'espèces } \\
\text { citées uniquement dans } \\
\text { les environs }\end{array}$ & 27,58 & 40,81 & 29,72 & 13,51 & 37,28 & 36 \\
\hline $\begin{array}{l}\text { Pourcentage par rapport } \\
\text { au total des espèces }\end{array}$ & 25,21 & 42,60 & 32,19 & 25,34 & 40,41 & 34,25 \\
\hline $\begin{array}{l}\mathrm{A}=\text { en augmentation, } \mathrm{B}=\text { en diminution, } \mathrm{C}=\text { disparues. } \\
\end{array}$ & & & & & & \\
\hline
\end{tabular}


Tableau 3: Espèces citées par la population riveraine non recensées dans le pré-inventaire par Sandjong (2012).

\begin{tabular}{llcc}
\hline$№$ & Noms vernaculaires (Mafa) & Noms scientifiques & Familles \\
\hline 1 & Gagad & Acacia albida* & Mimosaceae \\
2 & Gonokoud & Annona senegalensis* & Annonaceae \\
3 & Gangar & Borassus aethiopum & Palmaceae \\
4 & Lakalak & Daniellia oliveri $*$ & Caesalpiniaceae \\
5 & Katapalt & Entada africana* & Mimosaceae \\
6 & Pekelde & Ficus abutifolia* & Moraceae \\
7 & Mindek & Ficus dicranostyla* & Moraceae \\
8 & Goudav & Ficus gnaphalocarpa & Moraceae \\
9 & Kouzlar & Ficus sycomorus var. glumosa & Moraceae \\
10 & Gouvdad & Ficus platyphylla & Moraceae \\
11 & Tondaz & Haematophis barteri* & Anarcadiaceae \\
12 & Mawak & Kigelia africana & Bignoniaceae \\
13 & Mbalmbal & Maytenus senegalensis & Celastraceae \\
14 & Waf roua & Parkia biglobosa & Mimosaceae \\
15 & Foreu & Senna siamea & Caesalpiniaceae \\
16 & Chechem & Terminalia brownii & Combretaceae \\
17 & Wandar & Ziziphus mauritiana & Rhamnaceae \\
\hline
\end{tabular}

* espèces citées comme ayant disparu dans le parc.

Tableau 4: Estimation par intervalles de confiance de certaines opinions villageoises sur la gestion du parc.

\begin{tabular}{lccc}
\hline Opinion & $\begin{array}{c}\text { Nombre de } \\
\text { personnes } \\
\text { ayant répondu }\end{array}$ & $\begin{array}{c}\text { Pourcentage de } \\
\text { personnes } \\
\text { favorables }\end{array}$ & $\begin{array}{c}\text { Intervalles de } \\
\text { confiance au } \\
\text { risque 5\% }\end{array}$ \\
\hline $\begin{array}{l}\text { Observation du prélèvement des } \\
\text { ressources }\end{array}$ & 44 & 65,90 & {$[51,90 ; 79,90]$} \\
\hline $\begin{array}{l}\text { Besoins d'autorisations du } \\
\text { conservateur }\end{array}$ & 45 & 88,88 & {$[79,00 ; 99,00]^{*}$} \\
\hline $\begin{array}{l}\text { Besoins en ressources dans le } \\
\text { parc }\end{array}$ & 32 & 78,12 & {$[61,00 ; 90,00]^{*}$} \\
\hline $\begin{array}{l}\text { Acceptation du prélèvement des } \\
\text { ressources dans le parc }\end{array}$ & 30 & 73,33 & {$[58,00 ; 88,00]^{*}$} \\
\hline $\begin{array}{l}\text { Problèmes avec le service de la } \\
\text { conservation du parc }\end{array}$ & 35 & 48,58 & {$[32,02 ; 65,14]$} \\
\hline *Valeurs estimées à partir de l'abaque. & & & \\
\hline
\end{tabular}


Tableau 5: Récapitulatif des principales ressources prélevées ou dont le besoin a été exprimé au Parc National de Mozogo-Gokoro à l'issue de l'enquête.

\begin{tabular}{|c|c|c|c|}
\hline \multicolumn{2}{|c|}{ Type de ressources } & $\begin{array}{l}\text { Noms vernaculaires } \\
\text { ou en Français }\end{array}$ & Noms scientifiques \\
\hline \multirow{12}{*}{\multicolumn{2}{|c|}{$\begin{array}{l}\text { Bois de chauffage ou de } \\
\text { service }\end{array}$}} & Nawaya (Md) & Anogeisus leiocarpus \\
\hline & & Giler (Mf) & Acacia ataxacantha \\
\hline & & Daik (Mf) & Dalbergia melanoxylon \\
\hline & & Ltaroum (Mf) & Balanites aegyptiaca \\
\hline & & Hawoun (Mf) & Diospyros mespiliformis \\
\hline & & Broum (Mf) & Tamarindus indica \\
\hline & & Wandre (Mf) & Ziziphus spina-christi \\
\hline & & Gagad (Mf) & Acacia albida \\
\hline & & Golomb (Mf) & Acacia senegal \\
\hline & & Kekirbe (Mf) & Dichrostachys cinerea \\
\hline & & Chechebe (Mf) & Celtis integrifolia \\
\hline & & Tsinad (Mf) & Khaya senegalensis \\
\hline \multirow{29}{*}{$\begin{array}{l}\text { Produits } \\
\text { Forestiers } \\
\text { Non } \\
\text { Ligneux } \\
\text { (PFNL) }\end{array}$} & \multirow{6}{*}{$\begin{array}{l}\text { Animaux } \\
\text { (gibier) }\end{array}$} & Porc-épic (Fr) & Hystrix cristata \\
\hline & & Gazelles de Thomson (Fr) & Gazella rufifrons \\
\hline & & Mangouste (Fr) & Ichmeunia longicauda \\
\hline & & Varan (Fr) & Varanus sp. \\
\hline & & Lièvre (Fr) & Lepus saxatilis \\
\hline & & Ecureuil fouisseur $(\mathrm{Fr})$ & Xerus erythropus \\
\hline & \multirow{11}{*}{$\begin{array}{l}\text { Plantes } \\
\text { fourragères }\end{array}$} & Haval (Mf) & Stereospermun kunthianum \\
\hline & & Dozouk (Mf) & Pterocarpus erinaceus \\
\hline & & Goudav (Mf) & Ficus gnaphalocarpa \\
\hline & & Ltaroum (Mf) & Balanites aegytiaca \\
\hline & & Gagad (Mf) & Acacia albida \\
\hline & & Kotsape (Mf) & Andropogon spp. \\
\hline & & Tsakitsak (Mf) & Prosopis africana \\
\hline & & Daïk (Mf) & Dalbergia melanoxylon \\
\hline & & Vava (Mf) & Lonchocarpus laxiflorus \\
\hline & & Kodzoli $(\mathrm{Fu})$ & Anogeissus leiocarpus \\
\hline & & Mbalmbal (Mf) & Maytenus senegalensis \\
\hline & \multirow{12}{*}{$\begin{array}{l}\text { Autres plantes } \\
\text { utiles ou } \\
\text { médicinales et } \\
\text { produits } \\
\text { naturels }\end{array}$} & Nawaya (Mf) & Anogeisus leiocarpus \\
\hline & & Ouree (Md) & Tamarindus indica \\
\hline & & Wandre (Mf) & Ziziphus spina-christi \\
\hline & & Ltaroum (Mf) & Balanites aegyptiaca \\
\hline & & Magali (Md)) & Commiphora pedunculata \\
\hline & & Leved (Mf) & Grewia flavescens \\
\hline & & Mayawad (Mf) & Dioscorea sp. \\
\hline & & Baobab (Fr) & Adansonia digitata \\
\hline & & Chechebe (Mf) & Celtis integrifolia \\
\hline & & Tsinad (Mf) & Khaya senegalensis \\
\hline & & Tsakitsak (Mf) & Prosopis africana \\
\hline & & Miel et cire $(\mathrm{Fr})$ & // \\
\hline
\end{tabular}




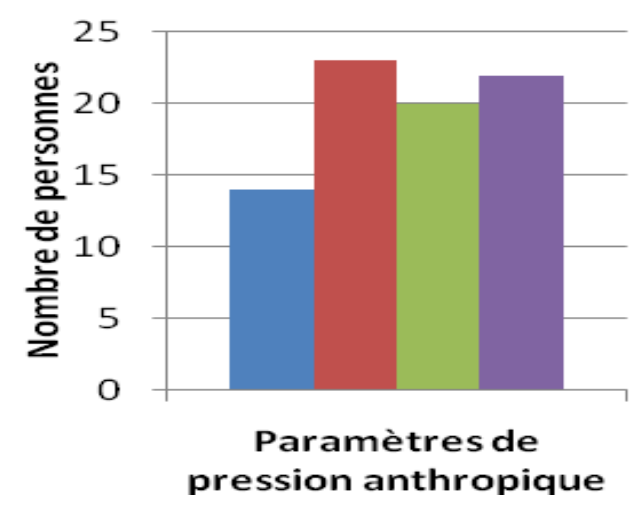

paturâge

coupe de bois

feux de

brousse

- presence de braconniers

Figure 2: Pression anthropique dans le parc.

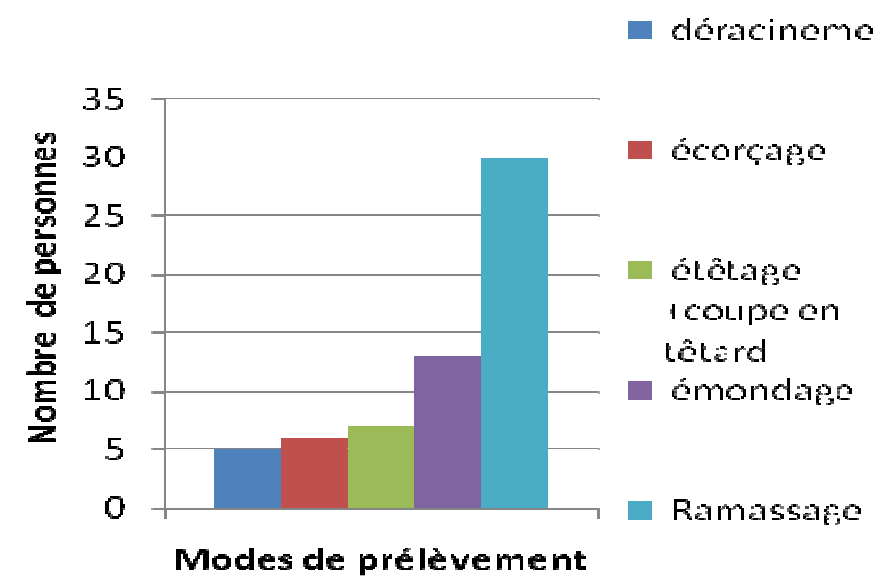

Figure 3: Modes de prélèvement de la ressource en bois.

\section{DISCUSSION}

L'évaluation des différentes activités des populations riveraines du parc annonce la forte pression actuelle et même future sur les ressources du parc, avec la croissance démographique, à travers la recherche de terres agricoles, de pâturages et les coupes de bois. Des auteurs (Dupuy et al., 1999 ; Wafo, 2008) soutiennent que cette prédisposition constitue une menace sérieuse pour la végétation, en plus de la sècheresse, des facteurs sociopolitiques, les difficultés scientifiques et techniques. Les femmes sont réputées être les principales utilisatrices de la ressource bois énergie et sont très impliquées dans la pratique de l'agriculture, le prélèvement des PFNL et la commercialisation du gibier. Le pourcentage faible de femmes observé $(8,88 \%)$ se justifie par le fait qu'avec le poids de la tradition, la plupart des femmes rencontrées ont préféré se rallier à la position de leurs maris, considérés comme chefs de familles. Avec l'âge moyen des personnes enquêtées $(49,7 \pm 8,4$ ans) et 
leur ancienneté dans les contrées riveraines (64\% supérieurs à 30 ans), le nombre de personnes âgées et anciennes dans les localités, susceptibles de mieux maîtriser les processus évolutifs ayant affecté le parc dans le temps, sa structure et sa superficie est convenable. La connaissance des limites du parc a été ainsi évaluée. Elle constitue l'un des principaux critères de prise de conscience de l'existence d'une aire protégée et d'évitement de son empiètement.

Toutefois, la limite de notre étude peut se révéler à travers le fait que plusieurs questions posées à certaines personnes enquêtées n'ont pas connu de réponses précises et parfois même pas de réponses du tout. Ceci est dû d'une part, à la peur de représailles, malgré toute la sensibilisation faite à ce sujet et d'autre part, certains jeunes n'ont pas suffisamment d'informations surtout concernant l'évolution du parc. C'est à croire que la tradition orale ne s'est pas suffisamment appesantie sur l'existence de cette végétation. En outre, un échantillonnage plus significatif et important, impliquerait de façon rigoureuse, la représentativité équitable de chaque groupe d'acteurs dans la population riveraine suivant l'activité et le sexe.

Concernant l'évolution spatiotemporelle de la structure de la végétation, Wafo (2008), en utilisant la télédétection, note une variation des éléments d'occupation du sol entre 1986 et 2001. Il observe d'une part, une augmentation des brûlis, de la forêt claire, de la forêt d'altitude, du sol nu peu couvert de végétaux et d'autre part, une diminution des zones de cultures, des herbacées et jachères, des savanes arborée et arbustive. Il faut ici affirmer pour nuancer, que son étude a intégré certaines zones limitrophes car, il estime la superficie du parc à 1723 ha.

Avec notre analyse du recul de la biodiversité animale et végétale, nous remarquons que les pourcentages d'espèces citées dans les réponses, et se trouvant uniquement dans les environs sont faibles, en raison de la dégradation de la végétation en ces lieux liée à diverses pratiques anthropiques. La disparition faunique peut s'expliquer par les migrations, la dégradation de l'habitat ou le braconnage (Dupuy et al., 1999). Wafo (2008) explique la réduction faunique par la petite superficie du parc et la proximité avec la population riveraine. Se rapportant au raisonnement de Cristofoli et Mahy (2010) cités par Diallo (2011) dans son analyse des effets combinés du climat et des pressions anthropiques sur la dynamique évolutive de la végétation d'une zone protégée du Mali, cette perte de biodiversité animale peut se justifier par la fragmentation de la végétation dans les environs du parc et son isolement. Les contraintes climatiques, le dynamisme compétitif des espèces ou la pression de l'Homme sont aussi des raisons justificatives. Dembélé (1996) affirme que l'impact des activités humaines est susceptible d'être à l'origine de modifications de la succession végétale et de dégradation du milieu. Le recensement des modes de prélèvement des ressources en bois est d'autant plus important que Bellefontaire (2005) a encouragé les acteurs des milieux forestiers à la recherche de techniques d'aménagement favorables à la régénération naturelle des ligneux. La disparition de certaines espèces animales disséminatrices peut expliquer la disparition ou la diminution de certains végétaux. L'importance des ligneux $(70 \%)$ parmi les plantes citées est une preuve de la forte interaction existant entre ces plantes et l'Homme et justifie les prémices de l'étude floristique préliminaire les concernant (Sandjong, 2012).

Au sujet de l'appréciation de la gestion et de l'utilité du parc, la coupe abusive de bois observée, est dévoilée comme étant un facteur important dans la dynamique régressive des ligneux (Faye et al., 2008 cités par Diallo, 2011). Une étude menée par Gautier et Ntoupka (2002), dans la région, indique cependant un changement de perception de la communauté des usagers (paysans, administrations et agents de développement) favorable à une inflexion de la dégradation des ressources arborisées dans la région. 
Malgré cette embellie perceptible, les aires protégées de la région subissent toujours des pratiques prohibées au regard de la loi avec des parcs arborés observés sans plan de gestion (Gautier et Ntoupka, 2002 ; Wafo, 2008). Les activités agricoles et piscicoles sont marginales sans grandes répercussions, tandis que le braconnage des ressources fauniques et les prélèvements de bois sont plus néfastes (Wafo, 2008). L'utilisation des ressources ligneuses prelevées correspond plus ou moins à leurs attributs déjà connus (Arbonnier, 2000). Pour remédier à l'appropriation illégale, plusieurs stratégies locales de gestion participative sont applicables dans la mise en œuvre des plans d'aménagement.

Les stratégies palliatives sont destinées aux populations riveraines et spécialement aux ouvriers des entreprises forestières par l'utilisation des sources alternatives de protéines animales (Mathot, 2003; ATIBT, 2005). Des aménagements associant les paysans, permettraient de concilier notamment la production par émondage de bois de feu, ressource rare, et la pérennité des parcs arborés (Smectala et al., 2005). Bergonzini (2004) propose des voies de réduction des pressions sur les ressources ligneuses et fourragères telles que l'amélioration du rendement énergétique des foyers, l'utilisation des énergies de substitution, la réduction des troupeaux et, le contrôle des techniques de gestion des pâturages et des feux. Au Parc National Oti-Ke'ran au Nord-Togo la vente du bois mort ramassé et l'affectation du revenu aux populations riveraines sont proposés aux gestionnaires pour faciliter la gestion participative (Adjonou et al., 2009).

L'éducation environnementale peut être pratiquée au sein des communautés locales des sites forestiers. Cette activité porte essentiellement sur la sensibilisation par voies de réunions faites au village, ou de discussions dans les groupes d'influence (FAO, 2001). Dans l'éducation formelle, les jeunes peuvent être influencés par des modes de pensée, une appréhension nouvelle de la nature, et une perception plus concrète de la préservation de l'environnement (MINEF, 1995). L'éducation interpelle également les médias qui, par le jeu de messages permanents, peuvent amener les populations à adhérer à l'idéal de la conservation de la biodiversité. Les actions répressives viennent généralement en dernier ressort. Elles sont prévues par la loi forestière camerounaise et appliquées dans le processus de suivi et de contrôle des activités forestières.

\section{REMERCIEMENTS}

Nos remerciements s'adressent à l'Ecole Normale Supérieure de l'Université de Maroua, Département de SVT qui a permis l'initiation et la validation de ce travail dans le cadre d'un Master, parcours Biologie des Organismes végétaux. Nous tenons également à manifester notre gratitude à l'endroit de toutes les personnes qui, par leur disponibilité ou leur participation, ont contribué au bon déroulement de l'enquête.

\section{REFERENCES}

Adjonou K, Bellefontaine R, Kokou K. 2009. Les forêts claires à Anogeissus leiocarpus du Parc national Oti-Ke'ran au Nord-Togo: structure, dynamique et impacts des modifications climatiques. Sécheresse, 20(4): 394-396.

ATIBT. 2005. Etude sur le Plan Pratique d'Aménagement des Forêts Naturelles de Production Tropicales Africaines. Application au cas de l'Afrique Centrale. (Volet 3). Aspects Faunistiques: Paris ; 99p.

Arbonnier M. 2000. Arbres, Arbustes et Lianes des Zones Sèches d'Afrique de l'Ouest. CIRAD-MNHN-UICN : Montpellier, France ; 541 p.

Bellefontaine R. 2005. Régénération naturelle à faible coût dans le cadre de l'aménagement forestier en zones tropicales sèches en Afrique. VertigO - la Revue Electronique en Sciences de l'Environnement, 6(2). 
Bergonzini J. 2004. Changements Climatiques, Désertification, Diversité Biologique et Forêts. SILVA, RIAT : 146 p.

Binot A, Joiris DV. 2007. Règles d'accès et gestion des ressources pour les acteurs des périphéries d'aires protégées. VertigO, la Revue Electronique en Sciences de l'Environnement, Hors-série 4.

Brabant P, Gavaud M. 1985. Les Sols et les Ressources en Terres du Nord Cameroun: Cartes et Notice Explicative. ORSTOM ; Feuille 19.

COMIFAC/CEEAC. 2007. Programme d'Action Sous-Régional de Lutte Contre la Dégradation des Terres et la Désertification en Afrique Centrale (PASR / LCD - AC), COMIFAC/ CEEAC. 65p.

Dembélé F. 1996. Influence du feu et du pâturage sur la végétation et la biodiversité dans les jachères en zone soudanienne-nord du Mali, Cas des jeunes jachères du terroir de Missira (cercle de Kolokani). Thèse de Doctorat, Université de Droit, d'Economie et des Sciences, Aix-Marseille III, 181p.

Diallo H, Bamba I, Sadaiou Y, Barima S, Visser M, Ballo A, Mama A, Vranken I, Maiga M, Bogaert J. 2011. Effets combinés du climat et des pressions anthropiques sur la dynamique évolutive de la végétation d'une zone protégée du Mali (Réserve de Fina, Boucle du Baoulé). Sciences et Changements Planétaires/ Sècheresse, 22(2): 97-107.

Donfack P, Seiny Boukar L, M'Biandoum M. 1996. Les caractéristiques du milieu physique. Actes de l'atelier d'échanges sur le thème : Agriculture des savanes du Nord Cameroun. CIRA : Cameroun ; 29 42.

Dupuy B, Maître HF, Amsallem I. 1999. Techniques de gestion des écosystèmes forestiers tropicaux: état de l'art. FAO, $146 \mathrm{p}$.
ECODIT. 2008. Evaluation de la Biodiversité et des Forêts au Sénégal. USAID: Sénégal; p.10-100.

FAO. 2001. Programme de coopération technique. Bilan des expériences et modèle d'aménagement pour une gestion durable des forêts, Cameroun (bilan provisoire), $70 \mathrm{p}$.

Gautier D, Ntoupka M. 2002. Une inflexion dans la dégradation des ressources arborées au Nord Cameroun. Actes du colloque, Savanes africaines : des espaces en mutation, des acteurs face à de nouveaux défis, Garoua, Cameroun, Jamin JY, Seiny Boukar L (eds). Prasac, N'Djamena, Tchad - Cirad, Montpellier, France, $9 \mathrm{p}$.

Halidou D. 2000. Decentralization and local natural resources management in far North Cameroon. Proceeding of international conference on Management of Fragile ecosystems in the North of Cameroon: the Need for an Adaptative approach, Maroua, 13-16 Nov 2000, p. 141-150.

Letouzey R. 1985. Domaine sahélien et domaine soudanien. Notice de la Carte Phytogéographique du Cameroun. IRA : Toulouse ; 1-26.

Malzy P. 1954. Quelques plantes du NordCameroun et leur utilisation. Journal d'Agriculture Tropicale et de Botanique Appliquée, TI(56): 37p.

Mathot L. 2003. Étude Faune UFA 10-030 au Cameroun. Rapport technique, PALLISCO, $20 \mathrm{p}$.

MINEF. 1995. Plan National de Gestion de l'Environnement (Volet Education environnementale). MINEF: République du Cameroun; 1-90.

MINEF. 2000. Inventaire ornithologique de la réserve forestière du Mayo-Louti et du parc national de Mozogo-Gokoro. Rapport technique et synthèse de l'avifaune, club ornithologique du Cameroun, Birdlife International, République du Cameroun, 16 p. 
Nguinguiri J. 1998. Les approches participatives dans la gestion des écosystèmes d'Afrique tropicale, FORAFRI, p.1-40.

Nké Ndih J. 2008. Déforestation au Cameroun: causes conséquences et solutions. Alternatives Sud, 15(155): 156175.

Ntoupka M, Fotsing E, Boubaoua A. 2006. Etat de la réserve forestière de Laf et des zones riveraines. Le Flamboyant, 62 :1217.

Ntoupka M. 1999. Impact des perturbations anthropiques (pâturage, feu, et coupe de bois) sur la dynamique de la savane arborée en zone soudano-sahélienne du Nord du Cameroun. Thèse de Doctorat de l'université, Paul Valery-Montpellier III, $260 \mathrm{p}$.

SACART. 2008. Carte du Cameroun au 1/50000. Société Africaine de Cartographie, Mokolo 4d.

Sandjong SRC. 2012. Etudes écologique et floristique du Parc National de MozogoGokoro (Cameroun): prospections préliminaires pour sa conservation et son aménagement. Thèse de Master, Université de Maroua, Cameroun, 83p.
Smektala G, Peltier R, Sibelet N, Leroy M, Manlay R, Njiti CF, Ntoupka M, Njiemoun A, Palou O, Tapsou. 2005. Parcs agroforestiers sahéliens: de la conservation à l'aménagement. VertigO la Revue Electronique en Sciences de l'Environnement, 6(2).

UICN. 2009. Programme de l'UICN au Cameroun plan stratégique 2009-2012, UICN, $46 \mathrm{p}$.

WAFO T. 2008. Les aires protégées de l'Extrême-Nord Cameroun, entre politiques de conservation et pratiques locales, Thèse de Doctorat, Université d'Orléans, Pôle Universités Centre Val de Loire, France, 325 p.

Yann LH. 1998. Climatologie et Agroclimatologie de la Province Extrême Nord (P.E.N.) du Cameroun, préparation de L'atlas de la P.E.N., 31p.

Zwiderwijk Adri B, Wouter T. 2000. Agricultural intensification in the Koza plain: on going trends and possible futures. Proceeding of International Conference of Fragile Ecosystems in the North of Cameroon: the need for and adaptative approach, Maroua, CML, CEDC, 209-220. 